\title{
A Non Invasive Sensor System for the Screening of Obstructive Sleep Apnea Syndrome ${ }^{+}$
}

\author{
Giorgio Pennazza 1,*, Marco Santonico ${ }^{1}$, Simone Scarlata ${ }^{2}$, Simona Santangelo ${ }^{2}$, \\ Simone Grasso ${ }^{1}$, Alessandro Zompanti ${ }^{1}$ and Raffaele Antonelli Incalzi ${ }^{2}$ \\ 1 Unit of Electronics for Sensor Systems, Department of Engineering, \\ Campus Bio-Medico University of Rome, 00128 Rome, Italy; m.santonico@unicampus.it (M.S.); \\ s.grasso@unicampus.it (S.G.); a.zompanti@unicampus.it (A.Z.) \\ 2 Geriatrics, Unit of Respiratory Pathophysiology, Campus Bio-Medico University and Teaching Hospital, \\ 00128 Rome, Italy; s.scarlata@unicampus.it (S.S.); s.santangelo@unicampus.it (S.S.); \\ r.antonelli@unicampus.it (R.A.I.) \\ * Correspondence: g.pennazza@unicampus.it; Tel.: +39-06-2254-19465 \\ + Presented at the Eurosensors 2017 Conference, Paris, France, 3-6 September 2017.
}

Published: 7 August 2017

\begin{abstract}
Obstructive Sleep Apnea Syndrome (OSAS) diagnosis can be confirmed only after the polysomnography (PSG), a non-invasive examination requiring one-night hospitalization. Moreover, up to $45 \%$ of people undergone PSG actually are free from OSAS. A non invasive sensor system collecting exhaled breath and giving a fingerprint of its composition should be a valuable mean for the selection of patients amenable to polysomnography (PSG). Here the BIONOTE (a gas sensor array based on Quartz micro balances functionalized with anthocyanins) together with the Pneumopipe (a patented device for exhaled breath collection) have been used in a study including 136 subjects. BIONOTE was able to correctly discriminate controls with respect to diseased individuals and Chronic Obstructive Pulmonary Diseased (COPD) against OSAS patients.
\end{abstract}

Keywords: OSAS; gas sensor array; Quartz micro balance; COPD

\section{Introduction}

OSAS is an age-related disease, it is associated with cardiovascular and metabolic problems, and often it overlaps with COPD [1]. The analysis of the Volatile Organic Compounds (VOCs) in exhaled breath with a fingerprinting approach is not invasive at all. VOCs in exhaled breath has been used to discriminate COPD and OSAS patients with respect to control individuals [2,3]. Considering that exhaled breath contains a lot of information about the individual health state, the study of a population of geriatric patients affected by a spectra of different diseases, gives the possibility of testing breath-print in a complex setup reflecting the conditions of a real diagnostic scenario. This consideration is crucial for OSAS, because the gold standard for its diagnosis is the polysomnography, an examination which request a night of hospitalization and which often (in almost the half of the subjects undergoing this test) gives a negative outcome (meaning non-diseased).

\section{Materials and Methods}

In this study exhaled breath of Control, COPD and OSAS individuals has been collected on Tenax GR adsorbing cartridge via Pneumopipe (EP12425057.2) and thermally desorbed in BIONOTE sensor cell, composed of eight QMBs covered with eight different types of anthocyanins [4,5]. The measure chain from exhaled breath collection to VOCs analysis is represented in Figure 1. Exhaled breath composition can be influenced by a number of environmental and metabolic variables. In order to prevent these interferences, patient's breath sampling was performed at 
morning, with patients fasting and smoking free for at least $12 \mathrm{~h}$. All patients were requested to wash their mouth, without using toothpaste, before the procedure, and they were not allowed to take alcoholic or aromatic beverages.

The suspected diagnosis of OSAS was tested through hospital PSG (Somnomedics; GmbH, Randersacker, Germany). Clinical data have been registered in order to better define each group and Partial Least Square models were built to test breath patterns ability to correct discriminate these groups.

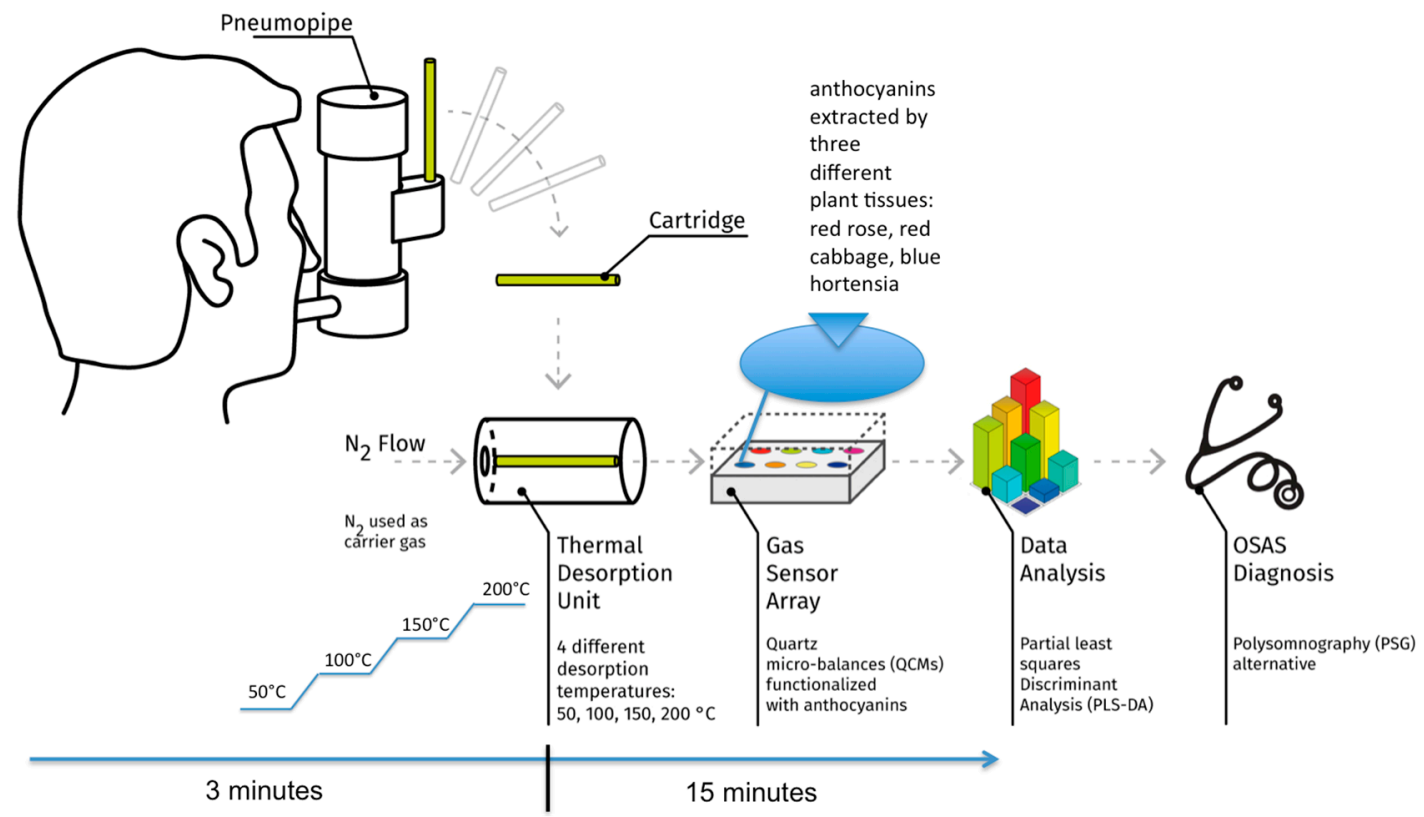

Figure 1. Measure chain of the sensor system: exhaled breath collection on a Tenax GR cartridge via Pneumopipe, thermal desorption of the cartridge content into the BIONOTE sensor chamber, BIONOTE measurement, data analysis and model elaboration (via PLS-DA crossvalidated with Leave one out).

\section{Results}

The main result is that control subjects have a distinctive VOCs pattern, which is different with respect to the patterns registered for the diseased ones, allowing a $100 \%$ correct classification. Moreover, BIONOTE has shown to be able to correctly discriminate hypoxemic $(60 \%)$ and non hypoxemic $(80 \%)$ OSAS patients, suggesting that the metabolic impact of hypoxemia contributes to generate a characteristic breath pattern. This last result suggests a peculiarity of VOCs pattern of COPD, hypoxemic and non hypoxemic OSAS patients, which indeed results in a good discrimination between COPD and non hypoxemic OSAS, with more ambiguity in the case of the hypoxemic OSAS diseased individuals, as shown in Table 1. It is very important to note that a clear discrimination between OSAS and COPD patients is not possible when considering a single cluster of OSAS individuals, including hypoxic and non hypoxic patients in a same class. Hypoxic patients plays an important role: none of them is identified as hypoxic, while the largest number of COPD patients not correctly classified are confused with Non-hypoxic OSAS patients.

Table 1. Confusion Matrix of the PLS-DA model discriminating among COPD and hypoxic and non hypoxic OSAS. The percentage of correct classification of each class is of $80 \%, 60 \%$ and $55 \%$ respectively for Hypoxic OSAS, non hypoxic OSAS and COPD.

\begin{tabular}{cccc}
\hline & Hypoxic OSAS & Non Hypoxic OSAS & COPD \\
\hline Hypoxic OSAS & 6 & 4 & 1 \\
Non hypoxic OSAS & 0 & 13 & 4 \\
COPD & 3 & 6 & 11 \\
\hline
\end{tabular}




\section{Discussion}

The results of this study support some important evidences: exhaled breath can allow discrimination of OSAS and COPD patients with respect to control individuals; hypoxemia plays a fundamental role in the identification of a specific breathprint, probably due to the great heterogeneity of OSAS patients. To this regard it is worth citing a recent study [3] by the same author of this work, which showed a cause-effect relationship between comorbidities and breathprint heterogeneity in OSAS patients. The controversial results obtained tell that the approach here proposed of considering more than one pathological conditions with respect to controls and among them, is strategic in order of finding out the real potentialities of such diagnostic technique in a real scenario. Looking at possible future developments, the increasing of population for a second experiment, should be focused on enlarging the two population (hypoxic and non-hypoxic) of OSAS patients, in order to better clarify the role of hypoxemia in the characterization of the breath-print for individual affected by respiratory diseases.

Acknowledgments: This study was partially funded by "Fondazione Roma" in the frame of the project "A novel approach to identify COPD phenotypes, forecast clinical course and plan the therapeutic strategy".

Conflicts of Interest: The authors declare no conflict of interest.

\section{References}

1. Sánchez, M.; Campos-Rodriguez, F.; Barbé, F. Obstructive sleep apnoea and cardiovascular disease. Lancet Respir. Med. 2013, 1, 61-72.

2. Greulich, T.; Hattesohl, A.; Grabisch, A.; Koepke, J.; Schmid, S.; Noeske, S.; Nell, C.; Wencker, M.; Jörres, R.A.; Vogelmeier, C.F.; et al. Detection of obstructive sleep apnoea by an electronic nose. Eur. Respir. J. 2013, 42, 145-155.

3. Antonelli Incalzi, R.; Pennazza, G.; Scarlata, S.; Santonico, M.; Vernile, C.; Cortese, L.; Frezzotti, E.; Pedone, C.; D'Amico, A. Comorbidity modulates non invasive ventilation-induced changes in breath print of obstructive sleep apnea syndrome patients. Sleep Breath. 2015, 19, 623-630.

4. Pennazza, G.; Santonico, M.; Incalzi, R.A.; Scarlata, S.; Chiurco, D.; Vernile, C.; D'Amico, A. Measure chain for exhaled breath collection and analysis: A novel approach suitable for frail respiratory patients. Sens. Actuators B Chem. 2014, 204, 578-587.

5. Santonico, M.; Pennazza, G.; Grasso, S.; D’Amico, A.; Bizzarri, M. Design and test of a biosensor-based multisensorial system: A proof of concept study. Sensors 2013, 13, 16625-16640. 